\title{
Magnetic resonance analysis of capillary formation reaction front dynamics in alginate gels
}

\author{
James E. Maneval, ${ }^{a}$ Diana Bernin, ${ }^{b}$ Hilary T. Fabich, ${ }^{c, d}$ Joseph D. Seymourc,d \\ and Sarah L. Codd ${ }^{d, e *}$
}

The formation of heterogeneous structures in biopolymer gels is of current interest for biomedical applications and is of fundamental interest to understanding the molecular level origins of structures generated from disordered solutions by reactions. The cation-mediated physical gelation of alginate by calcium and copper is analyzed using magnetic resonance measurements of spatially resolved molecular dynamics during gel front propagation. Relaxation time and pulse-field gradient methods are applied to determine the impact of ion front motion on molecular translational dynamics. The formation of capillaries in alginate copper gels is correlated to changes in translational dynamics. Copyright (c) 2011 John Wiley \& Sons, Ltd.

Keywords: pulsed-field gradient; gelation; dissipative dynamics; capillary formation; relaxation; alginate; magnetic resonance; MRI; NMR

\section{Introduction}

Alginate gels are formed by cation-mediated physical gelation of alginate composed of polysaccharide biopolymers of $\beta$-Dmannuronate and $\alpha$-D-guluronate. Alginate gels are produced in nature by both algae and bacteria. Plant-based alginate from brown algae is a source of food grade material that is broadly used in processed foods as a texture modifying filler. ${ }^{[1]}$ Microbial producers of alginate include Pseudomonas aeruginosa with different genetic variants of the microbe excreting molecularly different alginates, e.g. acetylated and deacetylated alginate. ${ }^{[2]}$ Of medical interest is a mutant strain of $P$. aeruginosa which plays a role in cystic fibrosis by producing a particularly thick alginate gel. Alginate gels are also of interest as a biomaterial for drug delivery and tissue engineering applications. ${ }^{[3-6]}$

Despite the prevalence of alginate gels in nature, industry, medicine and extensive research on them, the exact molecular mechanisms of physical gelation in these and other biopolymers is still not well understood. ${ }^{[7]}$ Enhanced understanding of the connections between the microscale molecular structure, the mesoscale network structure, ${ }^{[8]}$ and the macroscale material behavior or function has the potential to allow for controlled design of gels for specific applications. One aspect of the cation gelation of alginates is the formation of regular mesoscale structures in the form of ordered capillaries. ${ }^{[6,9,10]}$ This is of interest from the perspective of a biomimetic understanding of how to form structures in alginates for tissue constructs ${ }^{[4,6,11]}$ and understanding the thermodynamics and corresponding molecular dynamics of structure formation in biological systems in a general dissipative structure context. ${ }^{[10,12]}$ Toward this end, magnetic resonance (MR) microscopy has been applied to measure the spatially resolved water, spin-lattice relaxation $\left(T_{1}\right)$, spin-spin relaxation $\left(T_{2}\right)$ and molecular diffusion during the time-dependent heterogeneous reaction diffusion front formation of alginate gel. Significant MR studies on this type of gel have been conducted, ${ }^{[13-20]}$ but have not focused on capillary structure formation. Theoretical modeling of the interaction of cation transport and gelation ${ }^{[21,22]}$ in the absence of capillary formation have been undertaken. Models for capillary formation during cation-mediated gelation are far more limited, with a flow-induced dissipative structure model being the most well developed. ${ }^{[7,10,23]}$

This research builds upon and extends previous MR measurements to focus on reaction front dynamics and interprets the data in the context of current transport and reaction models. The organization of the article is as follows. In the Background section, we review past work on modeling and using MR to study the gelation process in alginates. The aim is to provide the basis for discussion of the results in the context of what is known about the phenomena associated with gelation so these ideas can be extended to capillary formation. After summarizing our Experimental Methods section, we discuss our findings for gels formed from calcium and copper ions and use those results to suggest improvements of existing models for gel formation in the Results and Discussion section.

* Correspondence to: Sarah L. Codd, Department of Mechanical and Industrial Engineering, Room 220 Roberts Hall, Montana State University, Bozeman, MT 59717-3800, USA.E-mail: scodd@coe.montana.edu

a Department of Chemical Engineering, Bucknell University, Lewisburg, PA 17837, USA

b Department of Chemical and Biological Engineering, Chalmers University of Technology, SE-412 96 Göteborg, Sweden

c Department of Chemical and Biological Engineering, Montana State University, Bozeman, MT 59717-3920, USA

d Center for Biofilm Engineering, Montana State University, Bozeman, MT 59717, USA

e Department of Mechanical and Industrial Engineering, Montana State University, Bozeman, MT 59717-3800 USA 


\section{Background}

\section{Models of the gel process}

Models of the alginate gelation processes are based on the concept that a divalent metal ion such as $\mathrm{Cu}$ or $\mathrm{Ca}$ interacting (reacting) with the guluronic residues of the alginate polymer in an aqueous solution creates a networked structure that physically gels the solution. The interaction can be expressed in terms of the reaction stoichiometry as

$$
\mathrm{MeCl}_{2}+2 \mathrm{NaAlg} \longrightarrow \mathrm{MeAlg}_{2}+2 \mathrm{NaCl}
$$

where Me stands for the metal ion. Further details on the chemistry of alginates and structures that can form when they are mixed with divalent ion are available in the review by Draget et al. ${ }^{[1]}$ and the original work of Thiele and Hallich. ${ }^{[9]}$

There have been two primary approaches to modeling the gel formation processes in alginates. The moving front model 25 assumes an infinitely fast reaction between ion and alginate to form a well-defined gel interface whose motion is controlled by the stoichiometry given above and the diffusivities of the reactants to the front. On the other hand, the continuum transport model ${ }^{[21]}$ couples a finite rate of gel formation to the diffusion of the reactants in both gel and solution phases. These models represent treatment of the gelation process as either a heterogeneous or homogeneous reaction process, respectively. ${ }^{[24]}$ While the moving front model has the advantage of being analytically solvable, the continuum transport model is potentially more flexible for modeling gelation processes. We present a summary of these models for use in discussion of the data.

The moving front model is expressed as a pair of partial differential equations that express the mass balance in each of the reactant phases (gel and solution)

$$
\begin{aligned}
\text { gel phase : } & \frac{\partial c}{\partial t}=D_{c} \frac{\partial^{2} c}{\partial z^{2}} \\
\text { alginate phase : } & \frac{\partial a}{\partial t}=D_{a} \frac{\partial^{2} a}{\partial z^{2}}
\end{aligned}
$$

In these expressions, $c$ and $a$ are the molar concentrations of the cation and alginate reactants, respectively, that form the gel and the diffusion coefficients $D_{c}$ and $D_{a}$ for each. These balances are coupled by a flux expression at the phase interface that includes both transport to, and reaction at the interface. Using the stoichiometry in Eqn (1), the flux continuity boundary condition relating the diffusion of cation and alginate at the moving front position, $z_{\mathrm{F}}(t)$, is

$$
\left.\left(-D_{\mathrm{c}} \frac{\partial c}{\partial z}\right)\right|_{z_{\mathrm{F}}(t)}=\left.\frac{1}{2}\left(D_{\mathrm{a}} \frac{\partial a}{\partial z}\right)\right|_{z_{\mathrm{F}}(t)}
$$

Details of the solution to this model are provided in Potter et al. [25]

The continuum transport, homogeneous reaction, model is succinctly expressed as a set of coupled partial differential equations based on the stoichiometry given above and species material balances in a homogeneous phase ${ }^{[21]}$ as

$$
\begin{array}{lc}
\text { gelling ion : } & \frac{\partial c}{\partial t}=D_{c} \frac{\partial^{2} c}{\partial z^{2}}-k_{11} c a^{2}-k_{\mathrm{g}} c a g \\
\text { alginate : } & \frac{\partial a}{\partial t}=\frac{\partial}{\partial z}\left(D_{\mathrm{a}} \frac{\partial a}{\partial z}\right)-2\left(k_{11} c a^{2}-k_{\mathrm{g}} c a g\right) \\
\text { gel : } & \frac{\partial g}{\partial t}=k_{11} c a^{2}+k_{\mathrm{g}} c a g .
\end{array}
$$

In these equations, $g$ is the molar concentration of gel, $k_{11}$ is the alginate and cation reaction rate and $k_{g}$ is the reaction rate for the addition of alginate to the existing gel molecular structures. In contrast to the moving front model, the continuum transport model does not define a discrete front where reaction occurs but rather allows for a balance of diffusion and reaction amongst the reactants to produce regions of strongly different composition (gel and alginate solution). Given a particular geometry along with an appropriate boundary and initial conditions, the solutions to this set of equations can be computed numerically. ${ }^{[17,21,26]}$ Reaction diffusion systems in which the reactants are initially separated provide a more controlled experimental system ${ }^{[27]}$ and are necessary for capillary formation. ${ }^{[9]}$ Both models may be applied to reaction diffusion systems with the difference being the presence of a discrete discontinuity at the gelation front where reaction occurs in the moving front model, in contrast to a reaction region of finite spatial extent in which product and reactant concentrations change continuously in space. The time dependence of the motion of the reaction front in a reaction diffusion system can be determined in both models, using a particular concentration of gel product or cation consumption to define a front location in the continuum transport model. The moving front model predicts the well-known scaling $z_{\mathrm{F}}(t) \sim\left(D_{\mathrm{c}} t\right)^{1 / 2}$ harkening back to the original work on interphase diffusion by Stefan. ${ }^{[24]}$

\section{MR studies of the gel formation process}

MR methods have been used extensively to study gels from both the polymer dynamics ${ }^{[28]}$ and gel reaction front perspectives. ${ }^{[13,14,17,18,25]}$ Relaxation and pulsed-field gradient methods ${ }^{[16,29]}$ have been used to study alginate solutions and the gels that are formed from them. Imaging of the gel formation process $^{[15,17,25]}$ has helped confirm, in a broad sense, that both the moving front and continuum transport models reasonably approximate the physical processes that occur during gelation. The front motion during alginate gelation by $\mathrm{Ca}$ has been shown to scale well with $z_{\mathrm{F}}(t) \sim\left(D_{\mathrm{Me}} t\right)^{1 / 2}$ indicating purely diffusive transport. ${ }^{[17,25]}$ In contrast, Potter et al. ${ }^{[15]}$ demonstrate that a modified front motion $z_{\mathrm{F}}(t) \sim\left(D_{\mathrm{Me}} t\right)^{1 / 2}+v^{*} t$ composed of a sum of the diffusive square-root in time and a linear in time scaling ${ }^{[30,31]}$ with a 'pseudoadvective' velocity $v^{*}$ provides a statistically significant better fit to the MR front motion data for cation diffusion into cartilage. The 'pseudoadvective' or 'Case II transport' behavior arises from stress relaxation due to polymer swelling in models of solvent diffusion into polymer. ${ }^{[30,31]}$

\section{Capillary formation issues and descriptions}

Most studies of alginate gels have focused on the use of ion solution at $0.1 \mathrm{M}$ or less, typically in the range of $30-50 \mathrm{mM}$. However, at higher ion concentrations (above $0.1 \mathrm{M}$ ) and for some ions (copper in particular), internal structures described as capillaries will form as the gel reaction takes place. ${ }^{[9,10]}$ These capillaries have been 


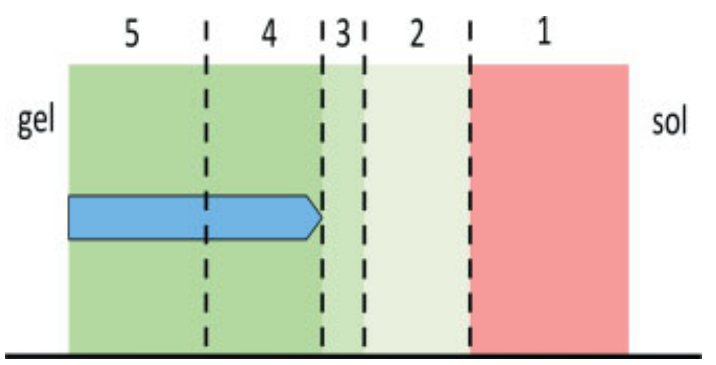

Figure 1. Proposed structural model of the gel front. The regions comprising the front are (1) sodium alginate solution, (2) dilute, pregel reaction zone where alginates react in small amounts with divalent ions, (3) homogeneous gel zone where the gel spans the length-scale of the sample, (4) capillary formation zone and (5) final heterogeneous gel formation zone.

used for the development of scaffolding systems and research has shown that fine control of the microstructure of alginate gels is possible. ${ }^{[3,5,6]}$ However, the mechanism for the formation of these capillaries has not been completely explained. The most welldeveloped current is that of Kohler and co-workers, ${ }^{[7,10,23]}$ where a Rayleigh-Bénard-like instability is used to explain the existence and propagation of the capillaries as gel formation progresses. An alternate theoretical perspective which can generate capillary-like structures in two-dimensions based on spinodal decomposition in the presence of anisotropic stress distribution in gels does not seem to have been applied to the alginate capillary formation previously. ${ }^{[32]}$

\section{A structural model of the front}

Alginate gels can display a wide variety of behaviors and the process whereby the gel is formed is an important factor in the nature and structure of the gel that is generated. A number of studies have examined the structures that form as a result of gelation of an alginate solution, ${ }^{[16,33]}$ but most of this work has focused on the structures that resulted from gelation and not on the molecular dynamics during structure formation. In the present study, we use MR measurements of the molecular dynamics occurring during gelation to generate a more detailed understanding of capillary formation in alginate gels. To help frame the discussion and interpretation of our data, we introduce a simple conceptual structural model of the gel front region in a sample based on our data (Fig. 1).

The structural model diagrammed in Fig. 1 posits the existence of five key regions in which the chemical and physical composition of the system changes from a uniform pure alginate solution (Region 1) to an ion-alginate gel (Region 5). Between the pure solute and gel regions there are three intervening regions. In the dilute gel region (Region 2), ion and alginate react to form gel species but at concentrations that do not lead to macroscopic gel networks that span the extent of the sample. Mikkelsen and Elgsaeter ${ }^{[21]}$ describe this concentration regime as the 'dilute regime' where the gel species are below some critical concentration level. ${ }^{[34]}$ Region 2 is considered a fluid region though the presence of an increasing level of gel in the region implies that the viscosity of the region would be changing quite rapidly but sample spanning clusters which arrest fluid-like motions are not yet present. ${ }^{[34-36]}$

At a certain point, the gel concentration passes the critical level and a sample spanning gel forms (Region 3). Initially, the gel forms homogeneously across the sample but as further ion physical cross-linking adds to the gel network, the chemical and mechanical properties of the network evolve, and the initial homogeneous gel evolves spatial heterogeneity or structure (Region 4). In Region 4, the gel is largely defined in a macroscopic structural sense but continues to incorporate ions into the gel matrix until the capacity of the gel for the ion is reached. While the transition from Regions 3 to 4 may be difficult to specify exactly based on extent of gelation, the key difference between these regions for our discussion purposes will be the presence of spatial heterogeneities such as capillaries or material properties such as density or elastic modulus. Region 5 is the ion-saturated ion-alginate gel.

\section{Materials and Methods}

\section{Alginate and ion solutions}

A 2 wt\% sodium alginate solution was prepared by adding sodium alginate powder from a brown algae source in powder form (Acros Organics, Geel, Belgium) to a $0.1 \mathrm{M} \mathrm{NaCl}$ (Sigma Aldrich) solution under stirring. The mixture was stirred at $70^{\circ} \mathrm{C}$ until the alginate powder was completely dissolved and then stored in a refrigerator until use. Metal ion solutions of copper chloride $\left(\mathrm{Cu}(\mathrm{II}) \mathrm{Cl}_{2}\right)$ and calcium chloride $\left(\mathrm{CaCl}_{2}\right)$ (Sigma Aldrich) were prepared at $0.5 \mathrm{M}$ concentration. All solutions were prepared with nanopure water and all chemicals were used without further purification.

\section{Sample preparation}

MR tubes (inner diameter $11.4 \mathrm{~mm}$ ) were cleaned with ethanol before being coated twice with a thin layer of the alginate solution and the thin layer of alginate was dried onto the tubes in a $110^{\circ} \mathrm{C}$ oven for $1 \mathrm{~h}$. This ensured that the gel that formed during reaction between the metal ion solution and alginate solution adhered to the tube walls. ${ }^{[5,9]}$ The coated tubes were then filled with the alginate solution and stored overnight to allow degassing. A polystyrene annulus with an outer diameter of $9.5 \mathrm{~mm}$ and inner diameter of $5.3 \mathrm{~mm}$ was then placed on top of the alginate solution to stabilize the ion-alginate solution interface. Approximately $2 \mathrm{ml}$ of the $0.5 \mathrm{M}$ salt solution $\left(\mathrm{CuCl}_{2}\right.$ or $\left.\mathrm{CaCl}_{2}\right)$ was added with a pipette. After 2-5 min, the tube was gently placed in the MR probe to start the measurements.

\section{MR relaxation of $\mathrm{Ca}$ and $\mathrm{Cu}$ aqueous solutions}

Relaxation times were measured for a series of copper and calcium ion concentrations between 0 and $0.5 \mathrm{M}$ using the inversion-recovery method for $T_{1}$ values and the CPMG method for $T_{2}$ values. Results were fitted to the straight line expression

$$
R_{i}=\frac{1}{T_{i}}=A[\text { ion }]+B \quad i=1,2
$$

where the parameters $A$ and $B$ are the slope and intercept values, respectively, of the fit. $B$ is the $1 / T_{2}$ value for zero ion concentration. Values of the parameter $A$, referred to as the relaxivity of the ion, are summarized in Table 1 for both copper and calcium chloride solutions. The findings for copper are in agreement with those reported by Potter and McFarland. ${ }^{[14]}$

As is well known, ${ }^{[37]}$ copper is a much more effective relaxation agent than calcium for the protons in water. It is interesting to note though, that while copper has essentially the same effect on both $T_{1}$ and $T_{2}$, calcium has a differential effect with a substantially stronger effect on $T_{2}$ than on $T_{1}$. 
Table 1. Relaxivity values, $A,\left(\mathrm{M}^{-1} \mathrm{~s}^{-1}\right)$ for aqueous solutions of copper and calcium chloride in the concentration range $0-0.5 \mathrm{M}$

\begin{tabular}{|lcrr|}
\multicolumn{2}{c}{ Calcium chloride } & \multicolumn{2}{c|}{ Copper chloride } \\
\hline$T_{1}$ & $T_{2}$ & $T_{1}$ & $T_{2}$ \\
0.175 & 1.55 & 778 & 935 \\
\hline
\end{tabular}

\section{MR methods}

All measurements were performed on a $300 \mathrm{MHz}$ Bruker Avance III system using a 15-mm birdcage of coil on a Micro2.5 imaging probe with maximum gradient strengths of $1.48 \mathrm{~T} / \mathrm{m}$ in all three directions. To observe the gel formation process, a set of MR measurements was created and then repeated multiple times over the course of an experiment. The sequential set of measurements included a two-dimensional image, multiple one-dimensional density profiles with varying echo time (TE) and repetition time (TR) from which relaxation time information was determined and multidirectional slice-selective pulsed gradient spin echo (PGSE) measurements. The imaging and profile measurements allowed tracking of the motion of the reaction front in the $z$-direction while the PGSE measurements measured the molecular translational dynamics as the front passed through a fixed region (slice) of the sample. By fixing the location of the PGSE measurement, we were able to clearly measure the molecular translational dynamics preand post-reaction of the system at a location in space. Timings and pulse-sequence repetitions were adjusted so that the full set of measurements could be repeated every 10-18 min and hence provided both good quality measurements and maintained a sufficient time resolution of the gelation process. The set of MR measurements were repeated continuously over approximately $15 \mathrm{~h}$ for the reaction front to fully traverse the alginate solution.

Details of the specific set of measurements made on the gel reaction front are as follows:

- Imaging: A standard multi-echo (CPMG) spin-warp sequence was used to create each set of $16 x-z$ two-dimensional images (TE) from $11 \mathrm{~ms}$ to $16 \times 11 \mathrm{~ms}=176 \mathrm{~ms}$ ) necessary to generate the $T_{2}$ maps. The two-dimensional images were generated by exciting a 1-mm slice and using two averages, a TR of $1 \mathrm{~s}, 256$ points in the frequency encode direction (FOV $=20 \mathrm{~mm}$ ) and 96 points in the phase encode direction (FOV $=13 \mathrm{~mm}$ ). The nominal spatial resolutions in the images were $78 \mu \mathrm{m}$ in the $z$ (frequency encode) direction and $136 \mu \mathrm{m}$ in the $x$ (phase encode) direction. Acquisition times for density and $T_{2}$ images were 4 min or less.

The one-dimensional version of the standard spin-warp sequence was used to more rapidly and frequently obtain one-dimensional profiles. These experiments retained the 1$\mathrm{mm}$ slice in the $y$-direction, the 256 point frequency encoding in the $z$-direction and the $11 \mathrm{~ms}$ echo spacing. Because the $x$-direction was not phase encoded, these profiles represent an integration over this direction of the sample. The TR for the one-dimensional profiles was $1 \mathrm{~s}$ for the $T_{2}$ measurements but ranged up to $10 \mathrm{~s}$ when measuring $T_{1}$ (see below).

- Relaxation: The 16 echo two-dimensional images and onedimensional profiles described above were fit to the singleexponential expression $M_{0} \exp \left(-\mathrm{TE} / T_{2}\right)$ at each pixel or location to provide apparent ${ }^{[38]}$ (effective) $T_{2}$-values at each spatial point in the images. By changing the TR for the onedimensional profiles from 60 to $5000 \mathrm{~ms}$, a one-dimensional distribution of $T_{1}$ values were estimated from a fit of the expression $M_{0} \exp \left(1-\mathrm{TR} / T_{1}\right)$ at each location in the profile. Data sets for one-dimensional profiles used for fitting of $T_{1}$ and $T_{2}$ consisted of the same 16 TE values and 5 TR values. Total acquisition time for the series of onedimensional measurements needed for one-dimensional $T_{1}$ and $T_{2}$ estimation was typically less than $2 \mathrm{~min}$. For the repetition and TE values used in the measurements, the minimum valid measurable values for $T_{1}$ and $T_{2}$ are estimated to be 50 and $5 \mathrm{~ms}$, respectively.

- Translational dynamics: A PGSE sequence was used that selectively excited a $0.5-\mathrm{mm}$ thick $(x)$ slice in the sample perpendicular to the direction of front motion $(z)$, usually located about $1 \mathrm{~cm}$ from the initial alginate/salt solution interface. Experimental parameters used for this standard PGSE method were TE $=40 \mathrm{~ms}, \delta=2 \mathrm{~ms}, 10 \leq \Delta \leq 30 \mathrm{~ms}$, NA $=$ 2, 4096 acquisition points, $\mathrm{SWH}=5000 \mathrm{~Hz}$. The effective or apparent diffusion coefficient (ADC) was estimated from the slope of a plot of the echo magnitude versus $(\gamma g \delta)^{2}(\Delta-\delta / 3)$ (a Stejskal-Tanner plot) while velocities were estimated from the slope of a plot of the echo phase versus $\gamma g \delta \Delta . .^{[39,40]}$ Total time for all fids in a given direction and for a given value of $\Delta$ was about $2 \mathrm{~min}$. ADC and velocity measurements were made in directions perpendicular $x$ and parallel $z$ to the front motion using $10-20$ gradient values ranging from 0.02 to $0.36 \mathrm{~T} / \mathrm{m}$. This range of gradient magnitudes was selected to provide up to $70-90 \%$ attenuation in the signal amplitude for water so that the measurements are in the low $q$-space regime and do not measure the full propagator of motion, rather they characterize the mean square displacement or variance $<z^{2}(t)>$ and hence the effective diffusion coefficient or $\operatorname{ADC} D(t)=\left\langle z^{2}(t)>/ 2 t^{[41]}\right.$

\section{Numerical methods}

Image and data processing were carried out in Prospa (Magritek, Wellington New Zealand, version 2.2) and MATLAB (MathWorks, Inc., Natick, MA, USA, version 7.9). Solutions to the continuum transport model were implemented in MATLAB using the built-in partial differential equation solver. ${ }^{[42]}$

\section{Results and Discussion}

\section{Comparison of models}

Except for the measurement of the relaxivity of copper and calcium chloride solutions (Table 1), we have not created calibration curves that connect the NMR relaxation time measurements to the cation concentrations within the gel phase that the models predict. The work by Duez et al. ${ }^{[17]}$ show that generally there is a linear relation between species concentration (ion, gel and alginate) and relaxation time but, as will be shown below, there are reasons not to take this approach and let the dynamic phenomena, as expressed in the measured relaxation times, diffusivities and velocities speak for themselves. The transport models can, however, provide a good framework for interpreting the NMR measurements and so it is important to get a sense of what the models predict before discussion of the measurements.

The moving front and continuum transport models have some important differences in their predictions that are worth noting before comparing the predictions to the data. In Fig. 2(a) and (b)) the solutions to the models are presented for comparison. The values of parameters used in the computations for these figures, 

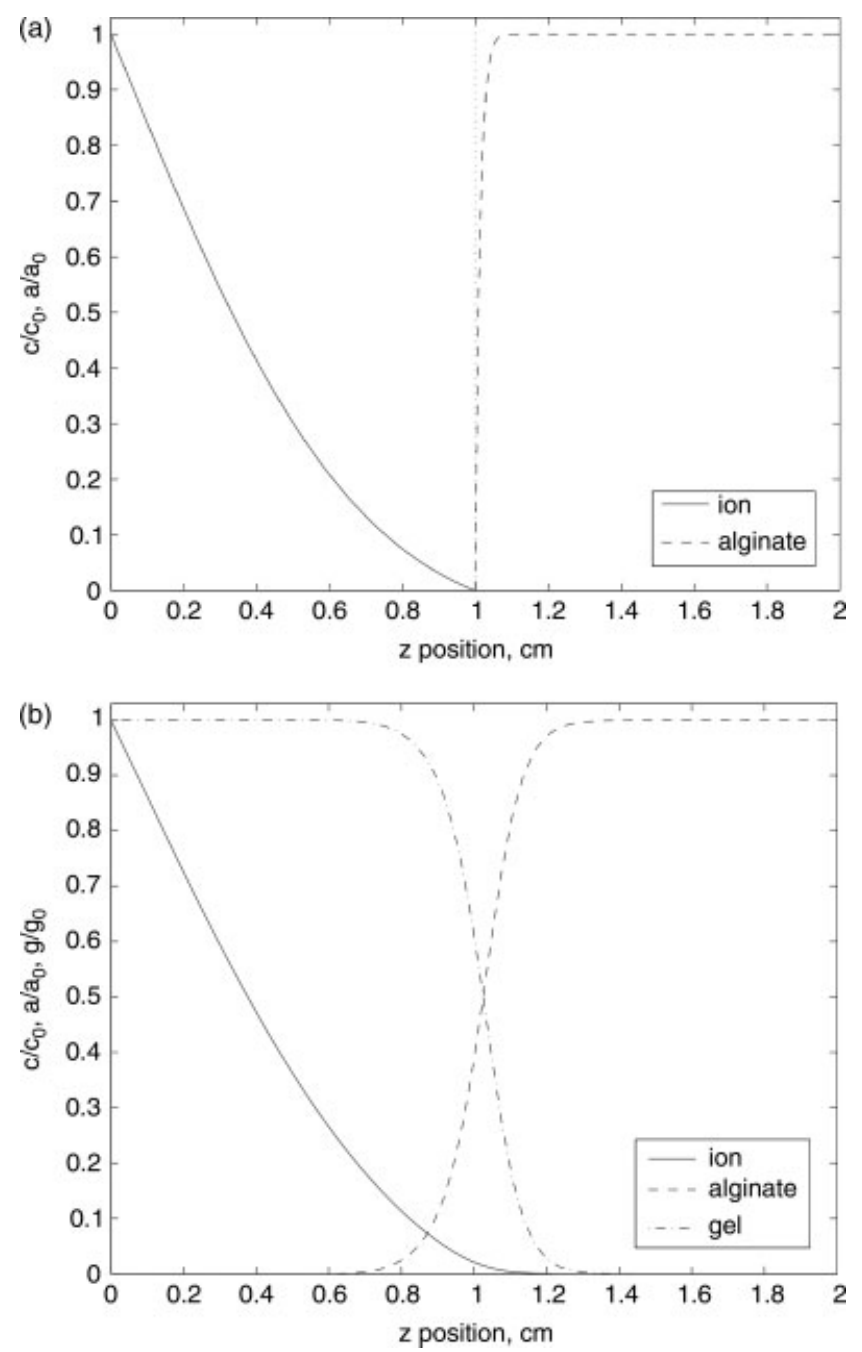

Figure 2. (a) Typical predictions of the moving front model. (b) Typical predictions of the continuum transport model.

e.g. diffusion coefficients, rate constants, were selected to be close to values reported in the literature but no attempt has been made here to use values that fit our measurements. Thus, the focus of Fig. 2 is to illustrate the qualitative differences in the two modeling approaches. The moving front model (Fig. 2(a)) generates a front through which neither alginate nor ion passes. At the front, the concentrations of both of these species are zero and, though the model does not explicitly mention it in the formulation, the concentration profile for the gel species is implied to be zero to the right of the front and a constant, non-zero value to the left of the front. These features make overall sense in the context of the gel formation process and the main strength of the model is that the motion of the front $z_{\mathrm{F}}(t)$ can be clearly predicted. ${ }^{[27]}$ However, when one considers the details of the predictions, there are some inconsistencies. For example, the decrease in the alginate concentration just ahead of the front without some sort of increase in the gel concentration implies that there is some sort of depletion layer close to the front. This depletion layer could be observable by the effects that a large change in alginate concentration would have on NMR parameters such as relaxation or diffusivity.

The continuum transport model (Fig. 2(b)) allows the two reactants to interpenetrate and, in doing so, loses an ability to clearly define a reaction front location. There are features of the concentration profiles predicted by this model that should affect NMR measurements. For example, if the location of the front is defined as the point when the alginate drops to half of its initial concentration, at $z \sim 1 \mathrm{~cm}$ shown in Fig. 2(b), then the ion concentration ahead of the front should have measurable effects on NMR relaxation times, especially if the ion is copper. Additionally, the slow rise of the concentration of the gel species, relative to the near instantaneous rise predicted by the moving front model, allows for introduction of different regimes of gel formation, i.e. dilute, critical or semi-dilute, to be evaluated and even incorporated into relaxation or diffusion models that aim to predict NMR parameters from species concentrations at a point in space and time. The direct mapping of measured NMR parameters to species concentrations predicted by the model is not possible, but the model predictions allow interpretation of the NMR measurements in the context of the species concentrations, thus generating data to test the model results.

\section{Imaging results}

Figure 3(a) presents typical water proton density and Fig. 3(b) $T_{2}$ images derived from the multi-echo image data sets acquired. The density images (Fig. 3(a)) show the clear difference in the relaxation effects of the ion used in the experiment. For the calcium experiment, the front location is marked by a very small change in the density due to uncorrected differences in $T_{1}$ between the solution and gel. This lack of contrast is an expected result because the proton density reported here corresponds to the density of water molecules in the sample. Differences in water density, or concentration, between the alginate solution, gel and ion solution for $\mathrm{Ca}$ as the reactive ion are not expected to be more than $2 \%$ of the value for pure water since the gels are $98 \%$ water. On the other hand, in the copper experiment, the gel region has significant signal loss and is essentially black, due to the strong paramagnetic relaxation properties of the copper ions in solution and the copper physically cross-linking the guluronic residues causing very short relaxation time values in the gel water protons. ${ }^{[37]}$

The copper image (Fig. 3(a)) reveals capillaries that are formed at the gel front and extend into the gel region. The calcium experiments did not systematically develop capillaries as the copper experiments did although a few larger capillaries did form in some runs. The higher signal from the capillary structures in the copper image of Fig. 3(a) is due to the fact that they contain water with levels of copper lower than those found either in the gel or in regions close to the copper solution source toward the top of the figure. While the water signal intensity is lower in the capillaries than it is in the alginate solution, the signal is still stronger than the water in contact with the gel phase suggesting that the gel phase is a very efficient relaxation agent.

Figure 4 shows detailed, high-resolution MR images of a sample after complete gelation with copper. The capillary structures generated in the alginate gel by the copper ions are clearly visible in both the longitudinal and cross-sectional image.

The bright region at the reaction front for the copper experiment proton density image in Fig. 3(a) is due to the differential relaxation effects of copper and alginate on the $T_{1}$ and $T_{2}$ of water. Alginate in the solution changes the $T_{2}$ of water more strongly than it does the $T_{1}$ of water. In the alginate solution just ahead of the gel front, low levels of copper estimated to be a distance of $50 \mu \mathrm{m}$ or less ahead of the gel front based on the relaxivity data of Table 1, cause more significant changes in the observed $T_{1}$ value than the $T_{2}$. Hence, for a given pulse sequence, TR material at or near 
(a)
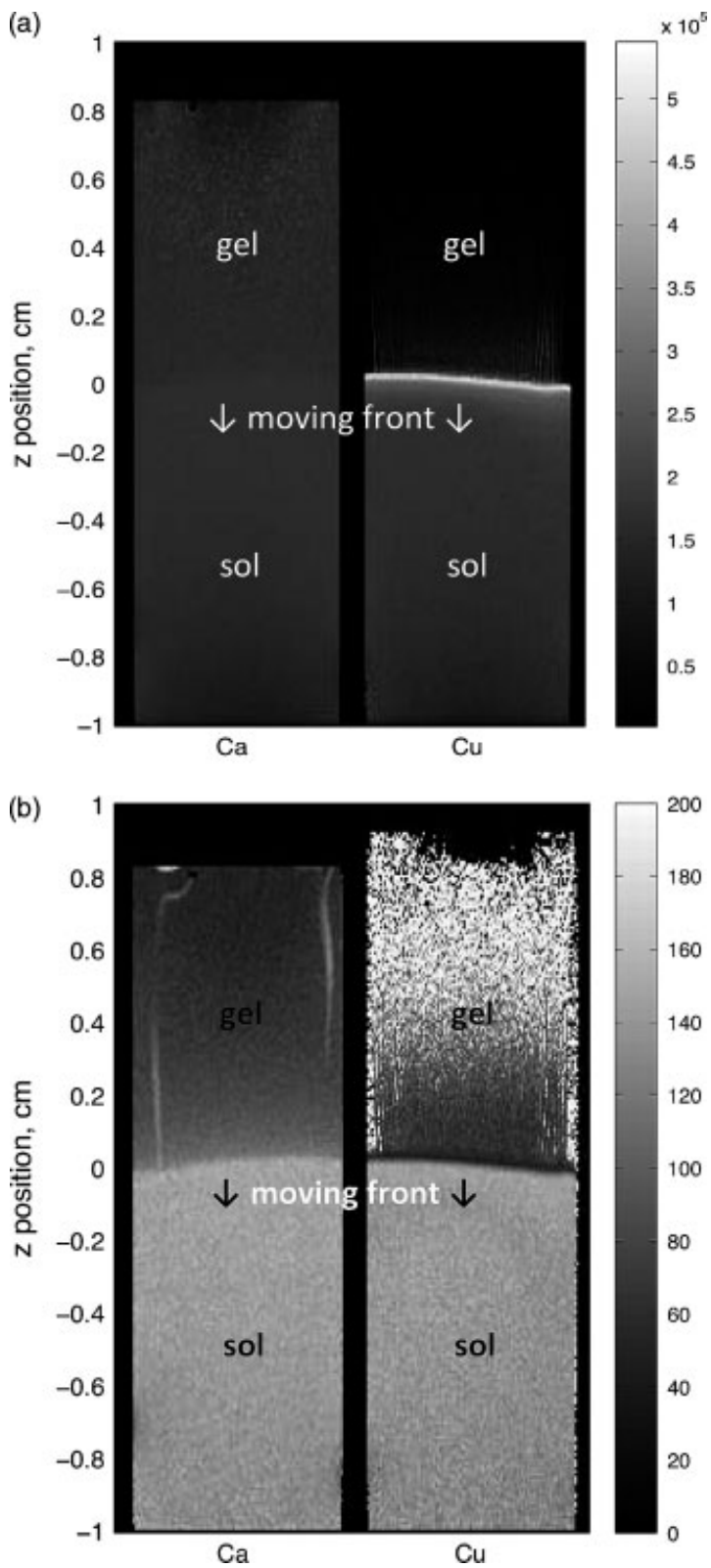

Figure 3. (a) ${ }^{1} \mathrm{H}$ density (top) and (b) $T_{2}$ (bottom, color bar in ms) images for experiments using either $100 \%$ calcium (Ca) (left, $t=312 \mathrm{~min}$ ) and $100 \%$ copper (Cu) (right, $t=485 \mathrm{~min}$ ) as the gelling ion. Times selected to show the gel front located in the region of the sample from which the PGSE measurements were made. Image resolution is $78 \mu \mathrm{m}$ in the $z$ (frequency encode) direction and $136 \mu \mathrm{m}$ in the $x$ (phase encode) direction.

the front will recover its equilibrium magnetization more quickly than material in the alginate solution leading to the appearance of a higher signal without change in the level of water. The $T_{2}$ images in Fig. 3(b) provide additional information about the gel formation process. There is a stronger $T_{2}$ contrast between the gel and alginate solution than there was for the density image for the calcium experiment. This makes it easier to distinguish alginate gel from alginate solution in the $T_{2}$ image. The similarity of the $T_{2}$ values in the capillaries seen in the figure reinforce the conclusions drawn from the density image in the copper experiment that the capillaries contain largely an aqueous solution of ions that is of different ion concentration from the gel that surrounds the

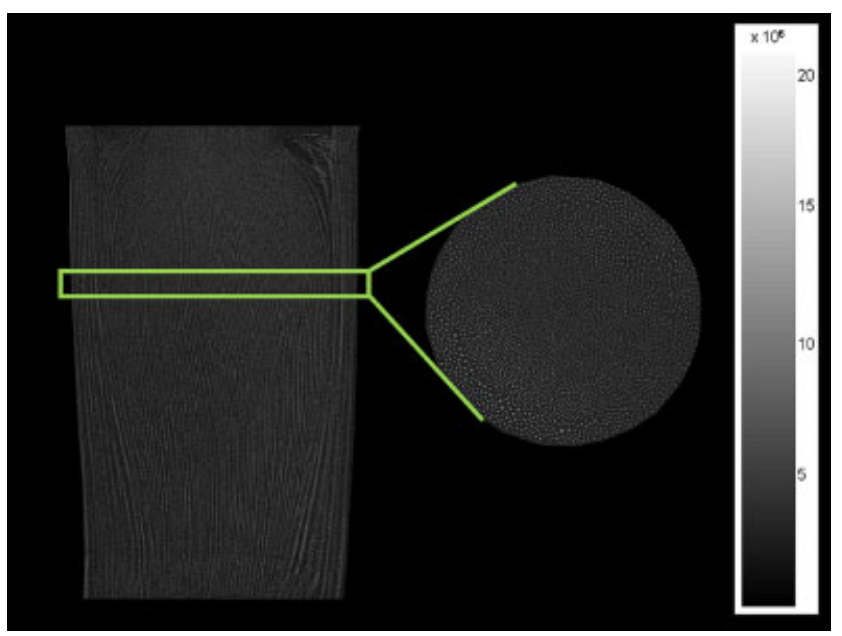

Figure 4. This higher resolution MR image clearly demonstrates the capillary structures formed after a $0.5 \mathrm{M} \mathrm{CuCl}_{2}$ solution had completely diffused through the $2 \%$ sodium alginate solution. These images were obtained after the gelation process had been completed in the entire sample. The longitudinal image has a resolution of $29 \times 39 \mu \mathrm{m}$ and a slice thickness of $1 \mathrm{~mm}$. The cross-sectional image has a resolution of $29 \times 29 \mu \mathrm{m}$ and a slice thickness of $0.5 \mathrm{~mm}$.

capillary. There is also a band of very low $T_{2}$ values located in the gel region just behind the bright band observed in the density image. This dark band will be discussed more fully in the next section.

\section{One-dimensional profiles}

As the images in Fig. 3 show, the gel formation process proceeds largely in one spatial dimension along the axial z-direction, so characterization of the process can be carried out efficiently and effectively by analyzing the one-dimensional axial $(z)$ profiles of NMR parameters. Figure 5 presents profiles of relaxation parameters reconstructed along with a representative raw data profile from the one-dimensional profile data taken close to the acquisition time of the $\mathrm{Cu}$ experiment images in Fig. 3. These profiles confirm the relative impact of copper in the gel and ion solution on the signal level and provide the spatial variation of the NMR measurements and structure of the gel front region that will help explain the structural model of the front that is presented in the Section on A structural model of the front.

All profiles in Fig. 5 show the same general trend from the alginate solution on the right corresponding to positions $z>0 \mathrm{~cm}$ in the reactor tube below the reaction front to complete signal loss in the gel/ion mixture on the left corresponding to positions $z<0 \mathrm{~cm}$ in the reactor tube above the reaction front. Some important differences and details are quantifiable in these profiles. Comparing the $T_{1}$ profile to the $T_{2}$ profile, we note that $T_{1}$ begins to decrease at dashed line A in Fig. 5 more than $1 \mathrm{~cm}$ ahead of the decrease in $T_{2}$. Only the presence of copper ions can cause a reduction of $T_{1}$ in this system and so the conclusion here is that the gelling ion is present ahead of the gel front but not at levels sufficient to cause a fully networked gel to exist in the sample. The ion and alginate form gel species but do so at a 'dilute' level. We note that this finding contradicts the fundamental assumption in the moving front model that neither reactant ion nor alginate gets past the gel front (Fig. 2(a)) but does lend support to the continuum transport model (Fig. 2(b)). 

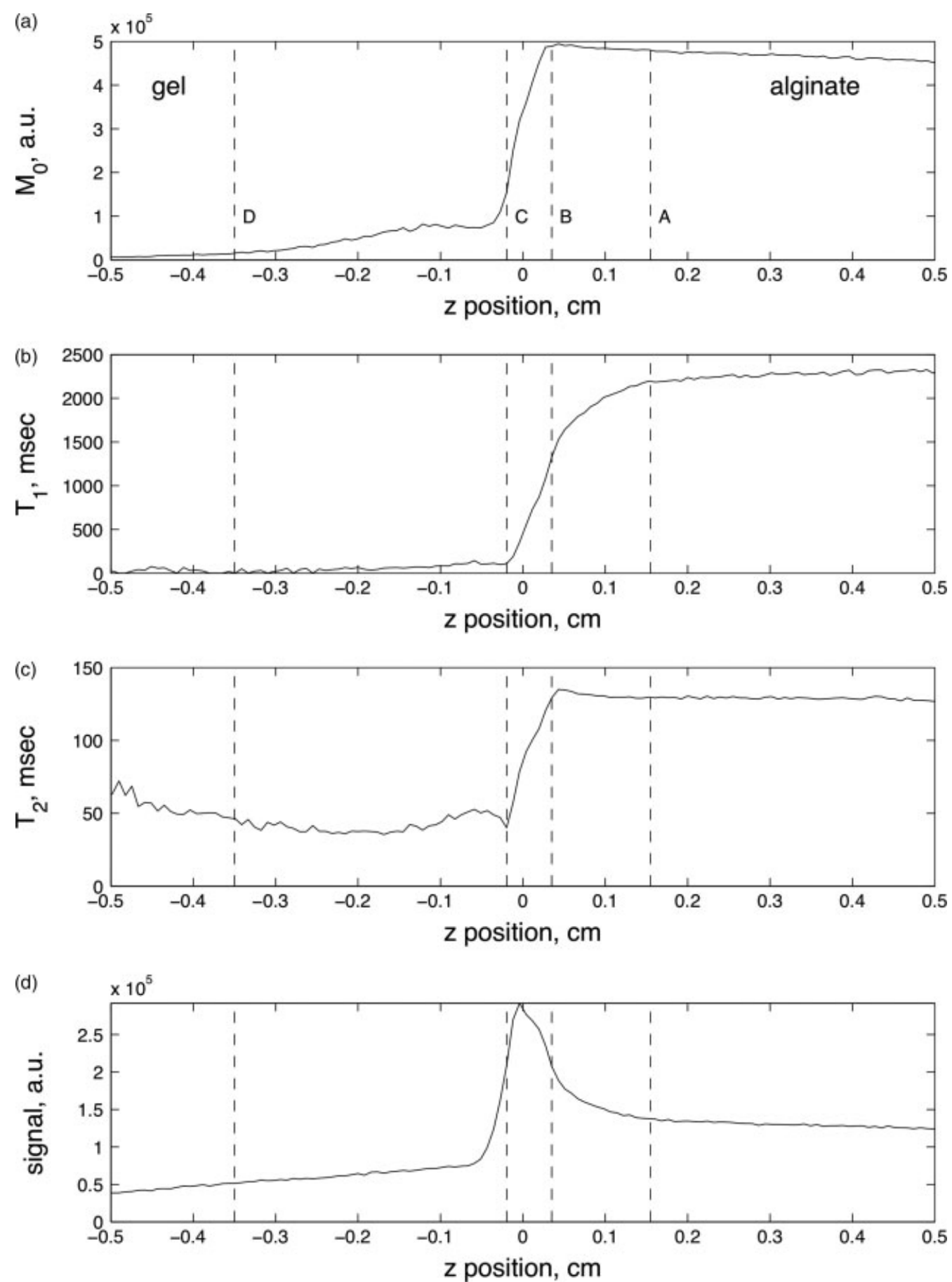

Figure 5. (a) Density $M_{0}$, (b) $T_{1}$ profile, (c) $T_{2}$ profile and (d) NMR signal for the first echo of the profiles with TE $=11 \mathrm{~ms}$, TR $=0.8 \mathrm{~s}$ for the $100 \%$ copper experiment acquired at $t=400 \mathrm{~min}$. Taken from the same experiment as the images shown in Fig. 3 .

Line $B$ at $z \sim 0.03 \mathrm{~cm}$ in Fig. 5 locates an important change in the $T_{2}$ and density profiles and a qualitative change in the slope of the $T_{1}$ profile. This point in the profile is interpreted to be the onset of the network gel in the sample associated with the transition from the dilute to the critical concentration of the gel species. For positions right of line $B, z>0.03 \mathrm{~cm}$ into the alginate solution portion of the sample, the density profile $M_{0}$ shows a relatively uniform value. For positions left of line $B z<0.03 \mathrm{~cm}$ into the gel portion of the sample, the density profile drops indicating that the signal from an increasing fraction of the water is not being faithfully included in the reconstruction of the density. Thus, line $B$ marks a critical level in the concentration of the gel species and a change in the nature of water relaxation within the gel matrix. This is consistent with the immobilization of copper ions in the evolving gel matrix enhancing the relaxation of the water protons through decreased mobility of metal ion and water molecules due to the gel formation. ${ }^{[37,43]}$

Between lines B and C, all relaxation parameters drop substantially as the gel enters the semi-dilute regime and forms a network that fully spans the size of the sample holder. At line $C$, 


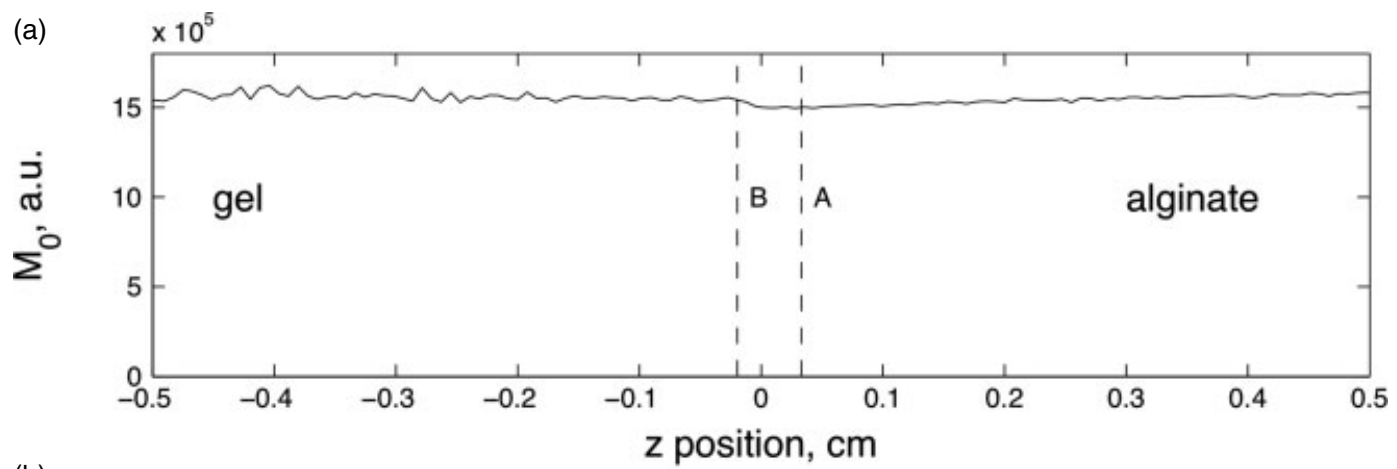

(b)
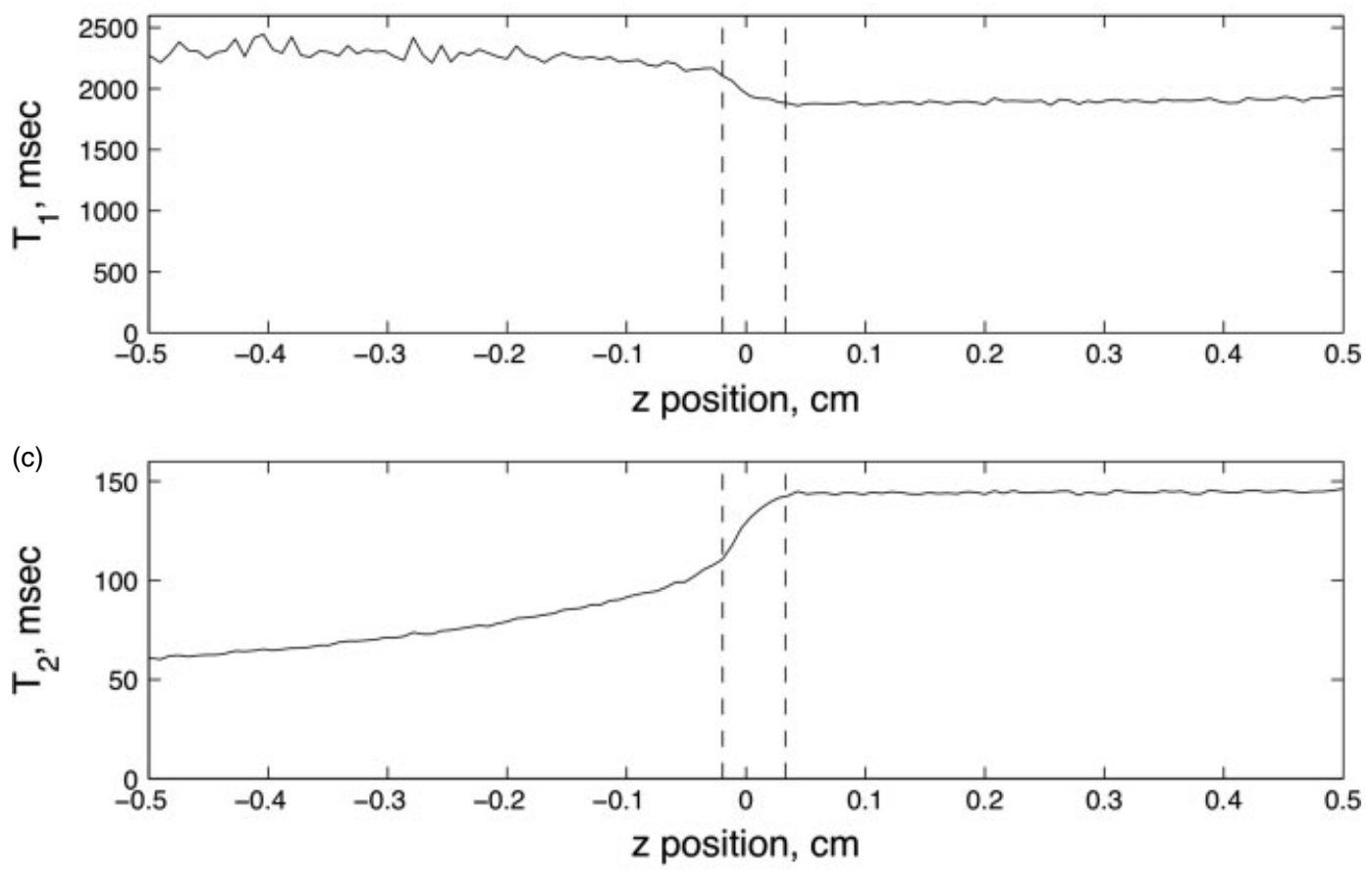

(d)

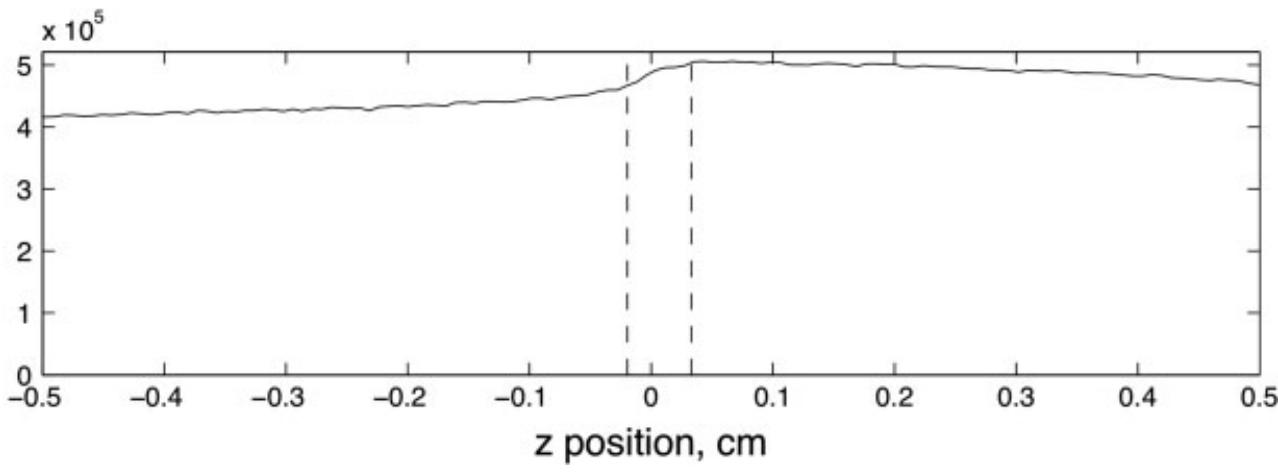

Figure 6. (a) Density $M_{\mathrm{o}}$, (b) $T_{1}$ profile, (c) $T_{2}$ profile and (d) NMR signal for the first echo of the profiles with TE $=11 \mathrm{~ms}$, TR $=0.8 \mathrm{~s}$ for the $100 \%$ calcium experiment acquired at $t=317 \mathrm{~min}$ Taken from the same experiment as the images shown in Fig. 3 .

the density and $T_{1}$ profiles become roughly constant while the $T_{2}$ profile exhibits a rebound to a level that then decays, along with the density and $T_{1}$ profiles, to the no-signal region marked by line $\mathrm{D}$ at $z \sim-0.35 \mathrm{~cm}$ above the front. The rebound is coincident with the dark band near the front that was noted above in the discussion of Fig. 3(a) for the $\mathrm{Cu} T_{2}$ map.

Figure 6 repeats the presentation in Fig. 5 for the $100 \%$ calcium experiment. As would be expected from the discussion of the density and $T_{2}$ images in Fig. 3, there is essentially no change in the density $M_{\mathrm{o}}$ going from the alginate to the gel and a much less dramatic change in $T_{2}$ compared to that for copper. The $T_{1}$ profile shows a small increase for values in the gel relative to the values in the alginate solution. Finally, the profile resulting from the first echo signal at $\mathrm{TR}=0.8 \mathrm{~s}$ does not show a maximum at the reaction front as the corresponding profile for copper in Fig. 5 does, a reflection of the lack of a bright band in the calcium images in Fig. 3. The profiles for the calcium experiment show little variation relative to the copper experiments largely due to the relaxivity 

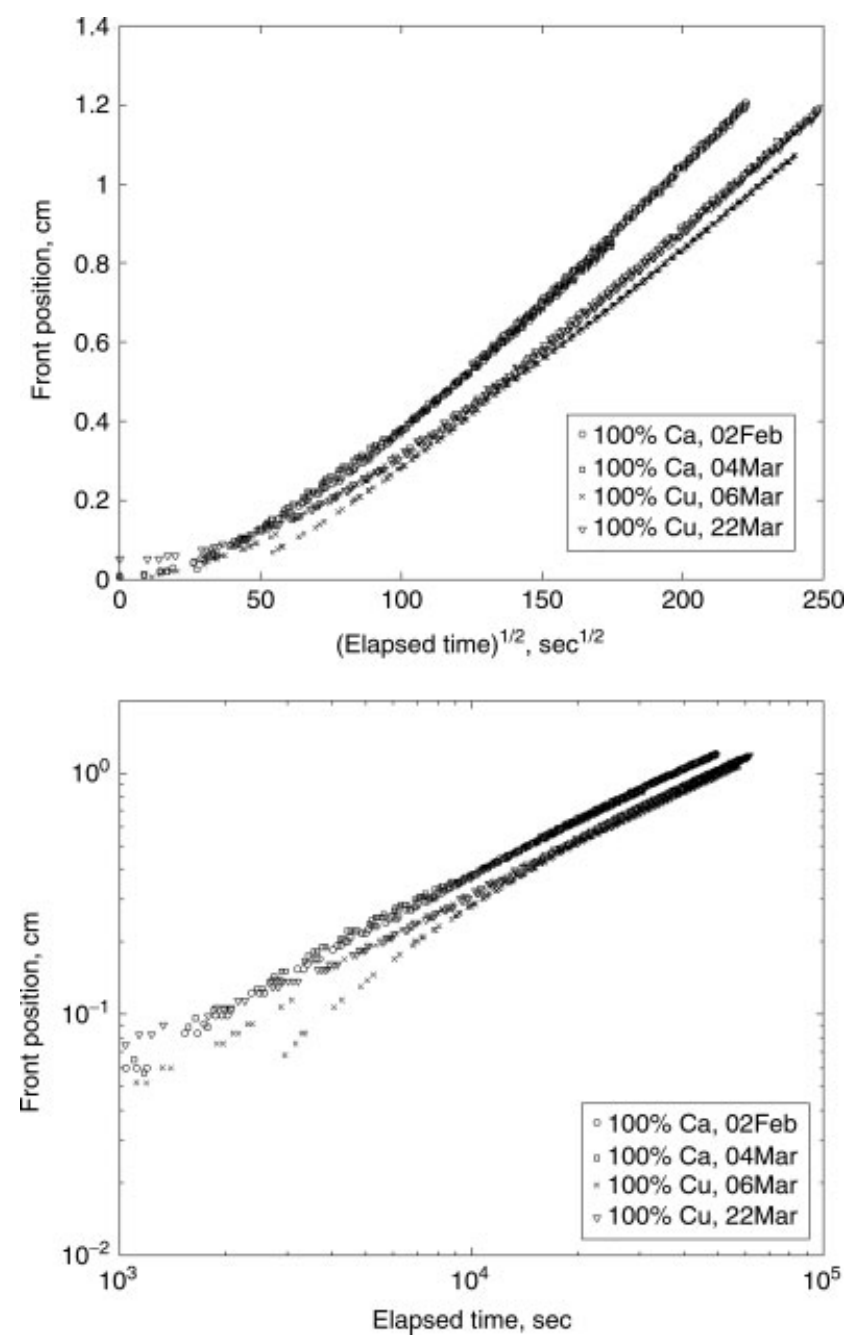

Figure 7. Front motion as a function of time $z_{\mathrm{F}}(t)$ for several representative runs of the gelation reaction for both $\mathrm{Ca}$ and $\mathrm{Cu}$ as the ion.

differences between calcium and copper (Table 1). However, the profiles do reveal some characteristics of the gel formation process. Line $A$ at $z \sim 0.03 \mathrm{~cm}$ in Fig. 6 locates the initial reduction of $T_{2}$ from its level in the alginate solution. Given the discussion of the copper results in Fig. 5, this is suggestive of the crossing of a critical concentration of the gel species and subsequent reduction of water rotational mobility. Line $B$ at $z \sim-0.02 \mathrm{~cm}$ marks a change in the slope of the $T_{2}$ profile that could be echoed by some of the changes in the $T_{2}$ slope between lines $B$ and $C$ in Fig. 5. Line B also appears to mark the end of the transition in the $T_{1}$ profile from the level in the alginate to the level in the gel. These observations suggest that there are multiple regimes in the gel formation process after the gel front passes. The further reduction in $T_{2}$ for $z<-0.02 \mathrm{~cm}$ indicates more restricted water rotational mobility caused by the gel network, in an aging or molecular rearrangement which reduces water mobility within the gel matrix.

The one-dimensional profiles directly provide measurement of the front motion $z_{F}(t)$ using a combination of the magnetic relaxation and signal intensity to define the front position at each acquisition time. Figure 7 shows $z_{\mathrm{F}}(t)$ measured for four repetitions of the gel reaction plotted as a function of $t^{1 / 2}$ as expected for a pure diffusion process and on a log-log plot. The pseudoadvective model $z_{\mathrm{F}}(t) \sim\left(D_{\mathrm{Me}} t\right)^{1 / 2}+v^{*} t$ fit to the data provides average values for the $\mathrm{Ca}$ reaction $D_{\mathrm{Ca}}=3.65 \times 10^{-5} \mathrm{~cm}^{2} \mathrm{~s}^{-1}, v^{*}=3.6 \times 10^{-6} \mathrm{~cm} \mathrm{~s}^{-1}$ while for the $\mathrm{Cu} D_{\mathrm{Cu}}=2.15 \times 10^{-5} \mathrm{~cm}^{2} \mathrm{~s}^{-1}, v^{*}=4.6 \times 10^{-6} \mathrm{~cm} \mathrm{~s}^{-1}$. The fits to the diffusion are very reproducible as evidenced by the linear regimes at long times $t^{1 / 2}>100 \mathrm{~s}^{1 / 2}$ in Fig. 7 while the velocity shows greater variability between reaction systems using the same cation with the Cu system having significantly larger pseudoadvective velocity. To further explore the differences between the time dependence of the front motion induced by the two cations the data is fit to a simple power law model $z_{\mathrm{F}}(t) \sim t^{n}$ where the power law index $n=1 / 2$ for normal diffusion and in general for random walks in fractals $n=1 / d_{\mathrm{w}}$ with $d_{\mathrm{w}}$ the dimension of the random walk which for Brownian motion or normal diffusion is $d_{w}=2 .^{[27]}$ The log-log data give fairly reproducible values of $n=0.57$ for $\mathrm{Ca}$ and $n=0.62$ for $\mathrm{Cu}$ indicating slightly stronger deviation from normal diffusion in the $\mathrm{Cu}$ reaction and consistent with a stronger influence of advective transport.

\section{Time course results: observations at a fixed position}

To investigate the molecular dynamics at the gel front PGSE measurements of effective diffusion (ADC) and velocity for the gel front reactions were made. As these measurements were not spatially resolved, it is useful for discussion purposes to generate non-spatially resolved relaxation measurements from the profiles in Figs 5 and 6 , by integrating the relaxation time profiles over the extent of the region selected for PGSE measurements. The images displayed in Fig. 3(a) and (b) show the position of the gel front as it passed through the region used to generate the PGSE data, a $0.5 \mathrm{~mm}$ region centered on $z=0$. This will allow a time-dependent analysis of the front as it passes the selected spatial region. The spatial average relaxation parameters are defined by

$$
\langle P\rangle=\frac{1}{l} \int_{-\frac{l}{2}}^{\frac{l}{2}} P(z, t) \mathrm{d} z \quad P=M_{0}, T_{1}, T_{2}
$$

where $I$, the length over which the spatial profile data is integrated, is the slice thickness of the non-spatially resolved PGSE data. In addition to monitoring the relaxation parameters time evolution through their spatially averaged values, it is useful to also monitor the total signal level in the selected region. There are several options for computing this quantity and the integral of the profile measured by the first $\mathrm{TE}\left(\mathrm{TE}_{1}=11 \mathrm{~ms}\right)$ such as that shown in the lower plot in Figs 5 and 6 is used. Thus, we define the average signal intensity in the selected region by

$$
\left\langle S_{1}\right\rangle=\frac{1}{l} \int_{-\frac{l}{2}}^{\frac{l}{2}} S\left(z, t ; T E_{1}\right) \mathrm{d} z
$$

Other measures could be used, for example the magnitude of the echo for the lowest gradient value used for the PGSE measurements, but the signal measured in Eqn (10) is most directly connected to the image intensity measurements associated with Figs 5 and 6.

Figure 8 presents the spatially averaged relaxation parameters and signal intensity as a function of elapsed time for a $100 \%$ copper experiment. The curves show the same features as the spatial profiles in Fig. 5, but are in a sense reversed left to right, due to the fact that the measurements are at a fixed location and 

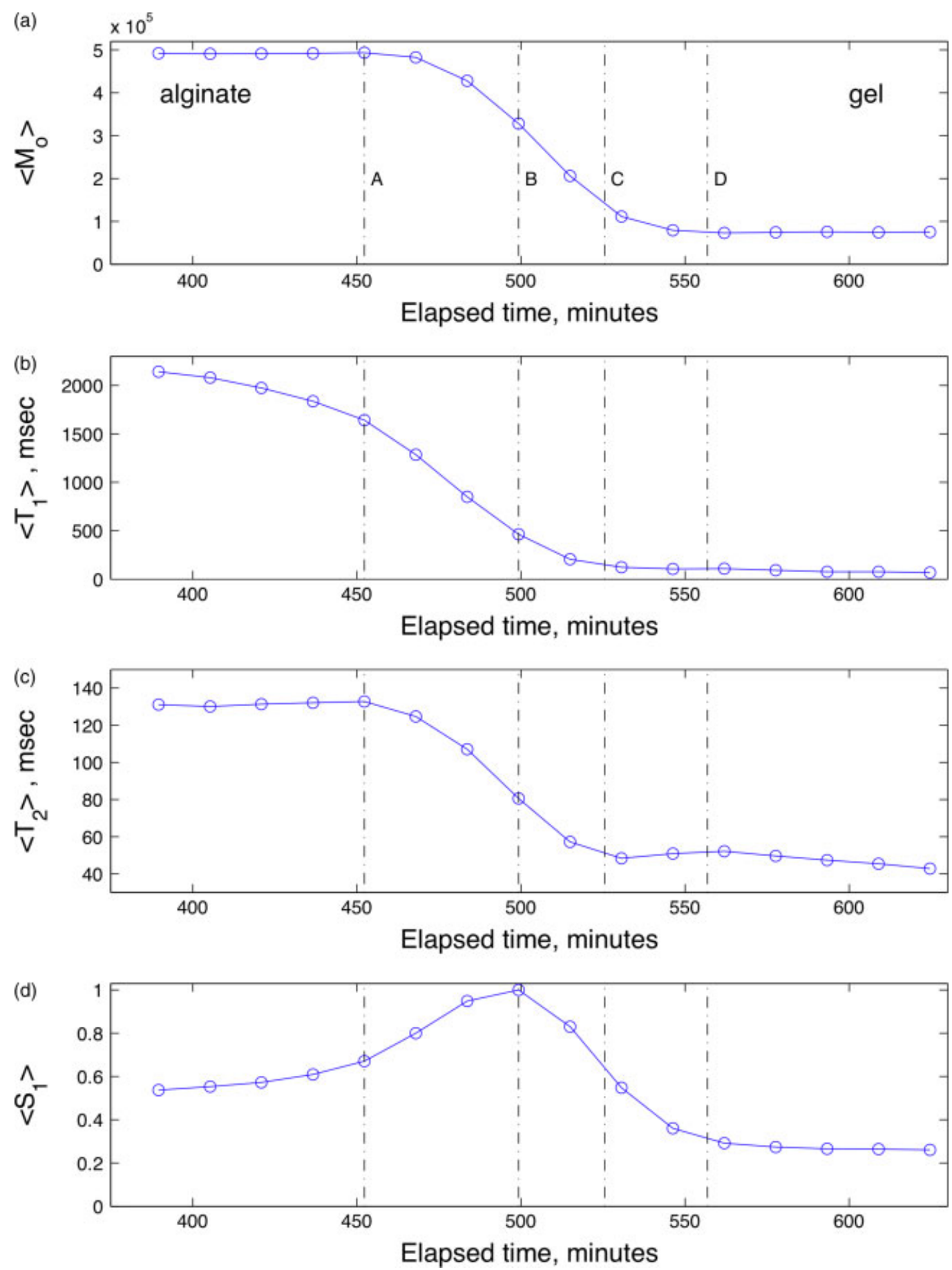

Figure 8. Spatially averaged image measurements of (a) density, (b) $T_{1}$ (c) $T_{2}$ and (d) first-echo signal intensity for the 100\% copper experiment over the region used to measure PGSE data.

early times to the left are prior to front passage. The time range shown has been zoomed in to focus on the passage of the gel front through the fixed spatial region. Line $A$ in Fig. 8 at $t \sim 450 \mathrm{~min}$ marks the entry of the critical gel region in the front as measured by the relatively sharp break in the $\left\langle T_{2}>\right.$ profile at that time. As can be seen from the $<T_{1}>$ profile, the dilute gel regime lasted for about 60 min prior to the onset of development of the critical gel in the slice. Line B marks the passage of the bright band (Fig. 3) through the region and is based on the maximum in the averaged signal intensity $<S_{1}>$ plot.

\section{Molecular translational dynamics}

Figure 9 presents measured ADC and velocity values from the region used in defining the averaged relaxation parameter values. Since both ADC and velocity values are obtained from the slope of a least square line through the data, the $90 \%$ confidence intervals on that slope value are used to bind the accuracy of the measured parameters. This confidence interval is presented as red lines parallel to the blue lines that connect the measurements. If the distance between the two red lines is small, there is very little 

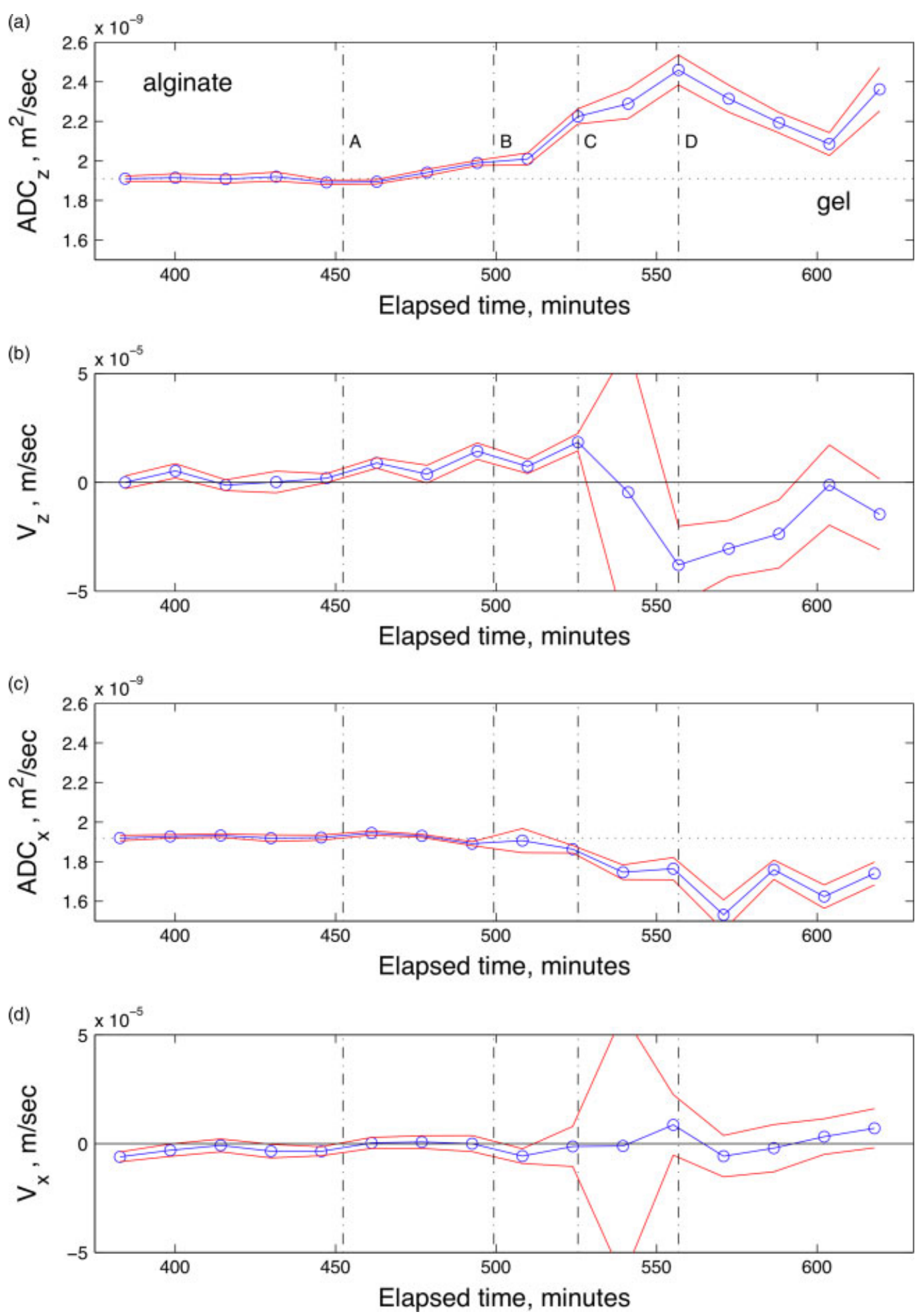

Figure 9. Measured axial $(z)$ and transverse $(x)$. (a) ADC in the axial direction $(z)$, (b) velocity in the axial (z) direction, (c) ADC in the transverse $(x)$ direction and $(\mathrm{d})$ velocity in the transverse $(x)$ direction for the selected region $(\Delta=20 \mathrm{~ms})$ for the $100 \%$ copper experiment. Dashed lines denote the extent of the confidence intervals on the slopes used to generate the parameter values (circles). For the axial velocity, positive values indicate motion toward the top of the sample.

dispersion in the data around the line that was fit to the data. The wider the distance between the red lines, the more noise and variability there is in the data. In the copper experiment, lines $C$ and $D$ in Figs 8 and 9 indicate significant changes in the PGSE data of Fig. 9. Specifically, line $C$ locates the time at which the noise in the measure of the axial $(z)$ and transverse $(x)$ velocities changes quite suddenly. Line $D$ locates the time at which the axial $A D C$ reaches a maximum value. The data in Fig. 9 provides the translational molecular dynamics during the passage of the gel front that complements the relaxation measurements in Fig. 5. 

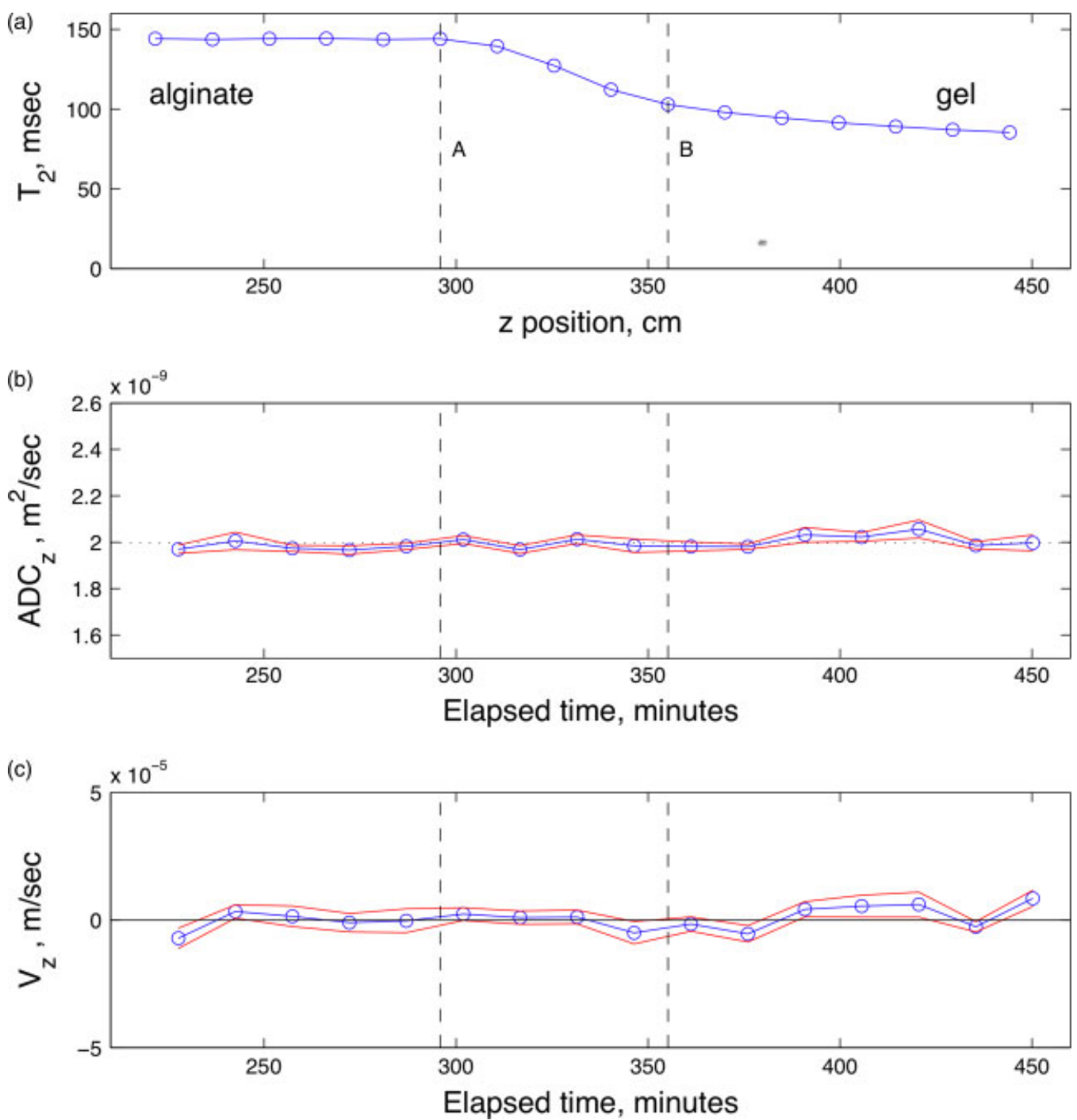

Figure 10. Measured (a) $T_{2}$ relaxation (b) ADC in the axial direction and (c) ADC in the axial direction for the selected region ( $\Delta=20 \mathrm{~ms}$ ) for the $100 \%$ calcium experiment. Red (gray) lines denote the extent of the confidence intervals on the slopes used to generate the parameter values (circles). For the axial velocity, positive values indicate motion toward the top of the sample.

Prior to the development of a gel phase (up to line A) diffusion in the alginate solution is isotropic. There is no translational motion other than the random motion of molecular diffusion water $D \sim 1.8 \times 10^{-9} \mathrm{~m}^{2} \mathrm{~s}^{-1}$ during this period. Between lines $A$ and $B 450$ min $<t<500$ min the relaxation data of Fig. 8 indicate the dilute gel regime has reached the slice of interest and the translational dynamics in Fig. 9 are similar to the alginate solution in magnitude. However, once a critical gel begins to develop as line B is approached, there is a small but significant upward trend in the axial velocity with a direction opposite that of the front motion and a slow increase in the axial ADC. The transverse velocity shows no change during the initial portion of the critical gel phase until it exhibits a strong increase in variability at line $C$ for $t \sim 525 \mathrm{~min}$ while the transverse to reaction front $x$-direction ADC reduces from the background value measured for the alginate solution.

The PGSE measurements in Fig. 9 suggest that as the gel front moves into a region there is molecular scale advective motion or velocity on the order of $10 \mu \mathrm{m} \mathrm{s}^{-1}$ induced in the axial direction opposed to the gel front motion. While such flows have been proposed as part of a model for capillary formation, ${ }^{[6,10,23]}$ the advective motion observed here is not compensated by significant transverse advective motion that this capillary formation by dissipative flow structure model requires as a consequence of the overall mass continuity equation. Rather, the axial advective dynamics are likely associated with the phase transition change in volume associated with the formation of the copper alginate gel. As indicated by the increase in the width of the statistical confidence interval on the axial velocity, the initially coherent induced advective motion eventually gives way to a broader distribution of motions that change on time scales that are smaller than the displacement observation time due to the gradient separation time $\Delta=20 \mathrm{~ms}$. This increase in confidence interval width suggests an increase in disorder in the microscale flow dynamics. This increase in dynamics that are random or incoherent relative to the $\Delta=20 \mathrm{~ms}$ displacement time scale enhances the axial ADC as would be expected for hydrodynamic dispersion where random diffusive motions interact with coherent advective motions. ${ }^{[41]}$ In the direction transverse to the motion of the front, the width of a zero-mean fluctuating velocity increases as the 
front passes between lines $C$ and $\mathrm{D}, 525 \mathrm{~min}<t<550 \mathrm{~min}$, reinforcing the idea that there are substantial increases in the randomness of the microscale flow field in the vicinity of the front. From the observed drop in the transverse ADC, these velocity fluctuations are also coupled to a more restricted diffusivity in this direction.

In Fig. 10, the combined PGSE and relaxation summary for the $100 \%$ calcium experiment is presented. In comparison to the copper results of Figs 8 and 9, the results in Fig. 10 show that the gel formation process for calcium at this concentration $(0.5 \mathrm{M})$ does not involve significant translational dynamics as measured by velocity or changes to the diffusion behavior in the sample. Line $A$ in Fig. 10 locates the entry of the gel front into the region from which PGSE measurements were obtained while line B attempts to identify the change of slope in the $T_{2}$ profile identified in the one-dimensional profiles in Fig. 6. There is no significant change in the PGSE measurements for alginate gelation due to calcium in stark contrast to the results for copper.

\section{Structural model revisited}

The results shown in Figs 8-10 can be used to support elements of the structural model for the gel front proposed in the Section on A structural model of the front (Fig. 1). The dilute gel region (Region 2), characterized by the formation of gel species under sufficiently dilute conditions that macroscopic measures of dynamics (flow, diffusion) are unchanged is invisible in the calcium experiment as none of the relaxation parameters are sensitive to changes in this regime. However, due to the strong relaxivity of the copper ion, there is clear evidence for this region in the changes in the $T_{1}$ values in this region for the copper experiment at times $<450$ min to the left of line A in Fig. 8.

Region 3, the initial gel region, is distinguished by the phenomena in the interval $450 \mathrm{~min}<t<525 \mathrm{~min}$ between lines $A$ and $C$ in Figs 8 and 9 and the interval $290 \mathrm{~min}<t<360 \mathrm{~min}$ between lines $A$ and $B$ in Fig. 10. In addition, the one-dimensional profiles of Fig. 5 for the copper experiment and Fig. 6 for the calcium experiment clearly show that the transition from Region 2 to Region 3 is a type of 'critical' transition with rapid changes in MR measured parameters as the gel spans the sample. ${ }^{[34-36,44]}$

Across this transition, both calcium and copper experiments show a distinct and rapid decrease in the measured $T_{2}$ value due to restricted rotational mobility of the water molecules as Region 3 is entered. For copper, the gel that forms in Region 3 gives way to a heterogeneous gel Region 4 where capillaries have formed from within the homogeneous gel. This is evident from the MR parameters between $525 \mathrm{~min}<\mathrm{t}<560 \mathrm{~min}$, lines $C$ and $D$ in Figs 8 and 9 where the slight rebound in $\left\langle T_{2}\right\rangle$ and significant changes in translational dynamics occur. The molecular dynamics indicate an increase in the rate of fluctuation of the advective dynamics measured by the velocity and strong anisotropy in the random translational dynamics measured by the ADC which increases in the axial direction and decreases in the transverse direction. The relaxation rates are characteristic of aqueous solutions of the ion with the total loss of the signal from the water in the gel portion of the sample. At times $t>560 \mathrm{~min}$, after line $D$, the gel continues to age (Region 5) but the increasing levels of copper in the water/capillary phase of the material prevent further observation of the process.

In contrast, for calcium there is no dramatic change in the molecular dynamic transport processes as Region 3 transitions to Region 4. As mentioned above, the relaxation parameters do exhibit a small change in Fig. 6 , to the left of line B but compared to the phenomena occurring when copper is used as the gelling ion, this is insignificant and the gel slowly ages as more calcium passes into the gel on its way to the gel front.

\section{Whence the capillaries?}

The MR data clearly indicate a difference in the translational molecular dynamics due to the formation of the heterogeneous structures in the form of capillaries in the copper system which are absent in the calcium system under our experimental conditions. The formation of capillary structures is dependent on many parameters including metal ion concentration and type, solution $\mathrm{pH}$, and alginate structure and concentration. ${ }^{[5,9,45]}$ The experimental conditions in this work consistently generated small ordered capillaries along the length of the gelled sample with copper and only generated limited large truncated capillaries in calcium. Such an effect is consistent with stronger binding by copper and increased gel strength relative to other metal ions. ${ }^{[45]}$ This stronger binding leads to the increased molecular dynamics during reaction shown in Figs 9 and 10 for the copper relative to the calcium system.

The measured advective translational dynamics or velocity are not consistent with the hydrodynamic convection roll model due to the lack of coherent transverse direction velocities. ${ }^{[7,10,23]}$ Rather the molecular dynamics measured indicate a spinodal decomposition mechanism for the capillary formation through a sol and gel phase separation. ${ }^{[32]}$ Capillary type structures have been predicted in two-dimension using a Ginzburg-Landau model for the polymer gel network volume fraction as the order parameter. ${ }^{[32,46]}$ The model is based on the total free energy containing an elastic stress contribution and a dynamic coupling between this stress and diffusion. ${ }^{[32]}$ Capillary-like structures are obtained in the presence of an anisotropic stress ${ }^{[32]}$ as is generated in the gel systems studied here due to the coating of the reactor tube walls with alginate and the unidirectional macroscopic front motion. The MR measured molecular dynamics in the copper system are highly anisotropic, consistent with an anisotropic elastic stress. Further measurements and analysis are required to make a more full quantitative comparison between MR data and spinodal decomposition models and this work is ongoing.

\section{Conclusions}

An extensive range of MR data measurement modalities provides further insight on the dynamics of gel formation and front propagation in the alginate ion systems. Relaxation data shows that the gel front has a definite structure that can be rationalized in terms of the continuum transport model proposed by Mikkelsen and Elgsaeter. ${ }^{[21]}$ Reaction front position and motion measurements suggest that the gel formation process is nonFickian and we find that the advective diffusive model ${ }^{[15,30,31]}$ fits the data well, with stronger advection in copper where capillaries are formed than in calcium where there is no or limited structure formation. PGSE measurements show that microscale flow processes near a gel front where capillaries form are complex and time varying. The measurements do not support the details of a proposed front propagation and capillary formation model based on dissipative flow structures. ${ }^{[7,10,23]}$ Rather, the measured anisotropic dynamics suggest further modeling efforts on this topic may more fruitfully pursue the spinodal-decomposition approach. ${ }^{[32]}$ 


\section{Acknowledgements}

Partial support from NSF CAREER awards CBET-0642328 (S. L. C) and CTS-0348076 (J. D. S) is acknowledged. Equipment funding was provided by the US NSF MRI Program and the M.J. Murdock Charitable Trust. J. E. M acknowledges support from Bucknell University sabbatical program. H. T. F was funded by INBRE Grant Number P20RR016455 from the National Center for Research Resources (NCRR), a component of the National Institutes of Health $(\mathrm{NIH})$. Its contents are solely the responsibility of the authors and do not necessarily represent the official view of NCRR or NIH.

\section{References}

[1] K. I. Draget, S.T.Moe, G. Skjacirck-Bræk， O. Smidsrød，Food Polysaccharides and their Applications (2nd edn), Taylor and Francis Group, LLC: Boca Raton, FL, USA, 2006

[2] D. E. Nivens, D. E. Ohman, J. Williams, M. J. Franklin, J. Bacteriol. 2001, 183, 1047.

[3] R. Dittrich, G. Tomandl, J. Am. Ceram. Soc. 2007, 90, 1703.

[4] A. Khademhosseini, J.P. Vacanti, R. Langer, Scientific American, 2009, pp. 64.

[5] F. Despang, A. Borner, R. Dittrich, G. Tomandl, W. Pompe, M. Gelinsky, Materialwiss. Werkst. 2005, 36, 761.

[6] B. J.Willenberg, T. Hamazaki, F.-W. Meng, N. Terada, C. Batich, J. Biomed. Mater. Res. A 2006, 79A, 440.

[7] H. Treml, S. Woelki, H.-H. Kohler, Chem. Phys. 2003, 293, 341.

[8] P.-G.de Gennes, Scaling Concepts in Polymer Physics, Cornell University Press: Ithaca, 1979.

[9] H. Thiele, K. Hallich, Kolloid Z. 1957, 151, 1.

[10] J. Thumbs, H.-H. Kohler, Chem. Phys. 1996, $208,9$.

[11] M. M. Stevens, H. F. Qanadilo, R. Langer, P. Shastri, Biomaterials 2004, 25, 887.

[12] I. Prigogine, Science 1978, 201, 777.

[13] K. Potter, T. A. Carpenter, L. D. Hall, Carbohydr. Res. 1993, 246, 43.

[14] K. Potter, E. W. McFarland, Solid State Nucl. Magn. Reson. 1996, 6, 323.

[15] K. Potter, R. G. S. Spencer, E. W. McFarland, Biochimica et Biophysica Acta 1997, 1334, 129.

[16] B. P. Hills, J. Godward, M. Debatty, L. Barras, C. P. Saturio, C. Ouwerx, Magn. Reson. Chem. 2000, 38, 719.

[17] J.-M. Duez, M. Mestdagh, R. Demeure, J.-F. Goudemant, B. P. Hills, J. Godward, Magn. Reson. Chem. 2000, 38, 324.
[18] N. Nestle, R. Kimmich, Colloid Surface 1996, 115, 141.

[19] N. Nestle, R. Kimmich, Biotechnol. Bioeng. 1996, 51, 538.

[20] N. Nestle, R. Kimmich, Magn. Reson. Imag. 1996, 14, 905.

[21] A. Mikkelsen, A. Elgsaeter, Biopolymers 1995, 36, 17.

[22] W. Barros, Jr. M. Engelsberg, J. Phys. Chem. A 2002, 106, 7251.

[23] H. Treml, H.-H. Kohler, Chem. Phys. 2000, 252, 199.

[24] R. B. Bird, W. E. Stewart, E. N. Lightfoot, Transport Phenomena (2nd edn), Wiley \& Sons: New York, 2002.

[25] K. Potter, B. J. Balcom, T. A. Carpenter, L.D. Hall, Carbohydr. Res. 1994, 257, 117.

[26] B. Thu, O. Gåserød, D. Paus, A. Mikkelsen, G. Skjåk-Bræk, R. Toffanin, F. Vittur, R. Rizzo, Biopolymers 2000, 53, 60.

[27] D. ben-Avraham, S. Havlin, Diffusion and Reactions in Fractals and Disordered Systems (1st edn), Cambridge University Press: Cambridge, 2000.

[28] J. P. Cohen-Addad, P. Huchot, A. Viallat, Polym. Bull. 1988, 19, 257.

[29] N. E. Simpson, S. C. Grant, S. J. Blackband, I. Constantinidis, Biomaterials 2003, 24, 4941.

[30] H. L. Frisch, T. T. Wang, T. K. Kwei, J. Polym. Sci. A-2: Polym. Phys. $1969,7,879$.

[31] N. A. Peppas, J. L. Sinclair, Colloid Polym. Sci. 1983, 261, 404.

[32] A. Onuki, S. Puri, Phys. Rev. E 1999, 59, R1331.

[33] C. Ouwerx, N. Velings, M. M. Mestdagh, M. A. V. Axelos, Polym. Gels Netw. 1998, 6, 393.

[34] H. H. Winter, in Structure and Dynamics of Polymer and Colloidal Systems, vol. 568 (Eds: R. Borsali, R. Pecora), Kluwer Academic: Dordrecht, Netherlands, 2002, pp. 439.

[35] L. Lu, X. Liu, L. Qian, Z. Tong, Polym. J. 2003, 35, 804

[36] X. Liu, L. Qian, T. Shu, Z. Tong, Polymer 2003, 44, 407.

[37] N. Bloembergen, L. O. Morgan, J. Chem. Phys. 1961, 34, 842.

[38] H. T. Edzes, D. van Dusschoten, H. van As, Magn. Reson. Imag. 1998, $16,185$.

[39] P. T. Callaghan, Principles of Nuclear Magnetic Resonance Microscopy, Oxford University Press: New York, 1991

[40] B. Blümich, NMR Imaging of Materials, Clarendon Press: Oxford, 2000.

[41] P.T. Callaghan, S. L. Codd, J.D. Seymour, Concept Magnetic Res. $1999,11,181$.

[42] R. D. Skeel, M. Berzins, SIAM J. Sci. Stat. Comp. 1990, 11, 1.

[43] M. Botta, Eur. J. Inorg. Chem. 2000, (3), 399.

[44] L. Lu, X. Liu, L. Dai, Z. Tong, Biomacromolecules 2005, 6, 2150.

[45] R. M. Hassan, M. T. Makhlouf, S. A. El-Shatoury, Colloid Polym. Sci. $1992,270,1237$

[46] N. Goldenfeld, Lectures on Phase Transitions and the Renormalization Group, Westview Press: Colorado, USA, 1992. 